\title{
Throughput Improvement Through Precoding in OFDMA Systems with Limited Feedback
}

\author{
Sameh Sorour, Amin Alamdar Yazdi and Shahrokh Valaee \\ Department of Electrical and Computer Engineering \\ University of Toronto \\ Toronto, Ontario, Canada M5S 3G4 \\ Email: \{samehsorour, ayazdi, valaee\}@ comm.utoronto.ca
}

\author{
Ronny Yongho Kim \\ Mobile Communications Technology Research Lab. \\ LG Electronics, Inc. \\ Anyang-shi, Kyungki-Do, Korea 431-749 \\ Email: ronnykim@lge.com
}

\begin{abstract}
In this paper, we study the possibility of throughput improvement through precoding in OFDMA based wireless systems with limited channel feedback. Precoding can increase the overall system throughput by selecting the use of higher modulation and coding schemes (if there are any) in each physical resource unit (PRU) under a maximum bit error rate constraint. For a specific form of precoding that we proposed in [1], we analytically derive a formula for the maximum PRU throughput. Sufficient conditions guaranteeing that our proposed technique outperforms the conventional technique are derived. Finally, numerical results show the amount of throughput gain achieved by our proposed technique.
\end{abstract}

\section{INTRODUCTION}

Future generation wireless systems, such as mobile WiMAX [2] and Beyond 3G (B3G) [3] networks, are expected to provide high speed wireless connectivity. Orthogonal Frequency Division Multiple Access (OFDMA), with a large number of subcarriers, has been chosen as the multiple access technique in these systems to achieve high rate wireless transmission. The choice of OFDMA arises due to its excellent performance in combating various wireless channel impairments such as multipath fading and inter-symbol interference. Moreover, OFDMA is very efficient in exploiting multiuser diversity by employing adaptive resource allocation [4]-[8] to different mobile receivers according to their instantaneous channel qualities.

Since resource scheduling in OFDMA wireless networks is performed at the base stations (BS), adaptive subcarrier allocation and bit loading techniques cannot be implemented in downlink without the knowledge of downlink channel qualities for all connected receivers. This necessitates the feedback of all this information to the BS, which results in a huge overhead. To reduce the amount of this feedback, current standards [2] [3] partition their spectral and temporal resources into physical resource units (PRUs) and allow receivers to report their channel qualities only once for the PRUs sharing the same subcarriers. In general, the subcarriers spanning both the spectral and temporal dimensions of the PRU are referred to as transmission units (TUs). To unify scheduling in both downlink and uplink, current standards assume that a PRU is the smallest allocatable unit. It must be assigned to only one

This work was sponsored in part by the LG Electronics Inc. receiver and only one modulation and coding scheme (MCS) is allowed to be used over all its TUs.

The MCS employed over the TUs of each PRU is determined from the average signal-to-interference-noise ratio (SINR) reported by the receiver that was granted this PRU, so as to satisfy the bit error rate requirement imposed by the network. If distinct symbols are sent over all the TUs (to which we will refer to as the conventional scheme), it is impossible to employ a higher MCS without violating the bit error rate constraint. The only way to use a higher MCS is then to reduce the number of distinct symbols transmitted in the PRU and introduce other alternatives to receive them. This procedure is generally referred to as precoding. In [9], [10], it has been shown that precoding can reduce the symbol error probability. Thus, we can employ precoding for any higher MCS to achieve a lower symbol error rate than the one achieved by the conventional scheme for the same MCS and SINR. Obviously, if the symbol error probability is reduced, the bit error rate will also decrease and can attain the desired level. At this point, two questions arise: Which precoding scheme should be employed? and would that lead to a throughput improvement?

The answer to the first question is obviously the precoding scheme that can achieve both the lowest symbol error reduction and an equal error probability to all transmitted symbols. We refer to the former property as the best improvement property and the latter as the symmetry property. The justification for the latter property is quite intuitive since it is necessary not to favor symbols over others in terms of error probability. The former property is justified by the fact that the stronger the ability of the scheme to reduce the symbol error probability, the lower the number of TUs needed to introduce redundancy and thus the higher the achieved throughput. In [1], we proved that a specific class of symmetric precoding schemes outperforms most other known symmetric precoding classes. In this paper, we will consider this precoding class and study its ability to improve the overall PRU throughput compared to the conventional scheme in such multicarrier environment with limited channel quality feedback. We show that we can indeed achieve throughput improvement for practical WiMAX and B3G systems using this precoding scheme proposed in [1].

The rest of the paper is organized as follows. Section II illustrates the system model. In Section III, we introduce 
the precoding class we explore in this paper. In Section IV, we derive an expression for the maximum PRU throughput achieved by this precoding scheme and sufficient conditions for that throughput to outperform the one achieved by the conventional scheme. Section V shows numerically the amount of throughput gains that can be achieved using our proposed technique. Section VI concludes the paper.

\section{SySTEM MOdEL}

Our model consists of a WiMAX or a B3G base station that employs OFDMA as the multiple access technology. Our focus is on downlink transmission but all our results apply to uplink transmission as well. The number of subcarriers in the cell can be as large as 2048 [2] [3] and even higher in newer standards. Each $N_{f}$ of these subcarriers are grouped together into subchannels. The subcarriers in each subchannel can be adjacent or distributed according to the subchannelization mode. Each subchannel is generally allocated to a receiver for $N_{s}$ symbols, which we generally term as PRU. Thus, each PRU includes $N=N_{f} \times N_{s}$ TUs. Current standards and prestandards set the value of $N_{f}$ between 10 and 25 subcarriers and the value of $N_{s}$ between 8 and 16 OFDMA symbols [2] [3], which makes practical PRU sizes $(N)$ in the range of 80 to 400 TUs.

The PRU allocation among different receivers is out of the scope of this paper. We only focus on the MCS determination for a PRU allocated to a given receiver. In current standards, the MCS is determined according to the SINR reported by the receiver, to which this PRU was granted, so as to transmit $N$ distinct symbols over the PRU TUs with a maximum threshold bit error rate $P_{b}^{t h}$. The number of bits in each symbol is determined by the spectral efficiency of the MCS employed in this PRU.

In the rest of the paper, we will use the following terminology and notation. The Spectral Efficiency (SE) of a MCS is defined as the effective number of information bits this MCS transmits on one TU. We define $b_{c}$ as the SE of the MCS allowed in the conventional scheme, $B$ as the set of MCSs whose SE are higher than $b_{c}$. Define $i$ as an index to the elements of $B, b_{i}$ as the SE of the $i^{\text {th }} \mathrm{MCS}$ in $B, R_{i}$ as the PRU throughput achieved by the $i^{\text {th }}$ MCS and $R_{\max }$ as the maximum PRU throughput achieved over all $i \in B$.

\section{Precoding Scheme}

Precoding has been introduced in the literature [9], [10] as a symbol protection scheme against fading conditions. In the context described in Section II, the idea of precoding can be introduced as reducing the number of distinct transmitted symbols in a PRU below $N$, and exploiting the remaining TUs in transmitting redundancy of these symbols. A symbol can thus be recovered through different alternatives which reduces the symbol error probability. One simple form of precoding is simple diversity in which the original symbols are simply repeated. In this case, the number of distinct symbols per PRU should be a divisor of the number of TUs. This diversity precoding is employed in several current standards. However, it is intuitively not suitable for the purpose of this study since a huge decrease in the transmitted distinct symbols will result as the employed diversity order is increased which will not lead to the desired throughput improvement.

Other forms of precoding can be obtained by performing finite field operations on the distinct data symbols. To explain this idea, assume that we send the $1 \times N$ vector $\underline{s}$ of coded symbols that is derived from the $1 \times U$ vector $\underline{u}$ of original distinct symbols $(U \leq N)$ as $\underline{s}=\underline{u} * \mathbf{A}$. In this case, $\mathbf{A}$ is a $U \times$ $N$ matrix that is generally referred to as the precoding matrix. The previous multiplication is performed over a finite field of suitable dimension. A non-zero entry $a_{i, j}$ in the precoding matrix determines the involvement of the original symbol $u_{i}$ in the coded symbol $s_{j}$. Note that the conventional scheme can be regarded as a special case of precoding where $U=N$, A is the $N \times N$ identity matrix and the SE is set to $b_{c}$.

In [1], we defined different classes of precoding matrices that satisfy the symmetry property, defined in Section I, and proved that a specific class of precoding matrices $\mathbf{A}^{*}$ achieves the lowest symbol error probability among these classes. The matrices of this class must include all the columns of the $U \times U$ identity matrix and the rest of the columns must be linearly independent vectors having no zero entries. In other words, any matrix of this class $\mathbf{A}^{*}$ must be a column permutation of the following matrix:

$$
\left(\begin{array}{cccccccc}
1 & 0 & \ldots & 0 & a_{1, U+1} & a_{1, U+2} & \ldots & a_{1, N} \\
0 & 1 & \ldots & 0 & a_{2, U+1} & a_{2, U+2} & \ldots & a_{2, N} \\
\vdots & \vdots & \ddots & \vdots & \vdots & \vdots & \ddots & \vdots \\
0 & 0 & \ldots & 1 & a_{U, U+1} & a_{U, U+2} & \ldots & a_{U, N}
\end{array}\right)
$$

where $a_{i, j} \neq 0 \quad \forall i \in\{1, \ldots, U\}, j \in\{U+1, \ldots, N\}$ and are chosen so that any $U$ columns of $\mathbf{A}^{*}$ are linearly independent. In [11], it has been proved that the aforementioned precoding matrix is also the optimum precoding matrix among all matrices having the symmetry property. Consequently, this is the best matrix that serves our purpose since the stronger the ability of the precoding matrix to reduce the symbol error probability, the lower the amount of redundancy needed for a MCS in $B$ to satisfy the bit error rate constraint. In the next section, we will provide an analysis of the maximum PRU throughput achievable by applying this precoding scheme on the MCSs in $B$ and derive the sufficient conditions needed for our scheme to outperform the conventional one in terms of PRU throughput.

\section{AnAlysis OF the PRU Throughrut}

\section{A. Derivation of the Maximum Throughput}

In this section, we aim to find an expression for the maximum throughput achieved by our proposed precoding scheme using $\mathbf{A}^{*}$. To make the problem general, precoding can be performed by either coding on all the $N$ TUs of the PRU or partitioning these TUs into $K$ subsets, each of size $F_{k}\left(\sum_{k=1}^{K} F_{k}=N\right)$, and employing precoding on each of them using $U_{k}$ symbols $\left(U_{k}<F_{k}\right)$. One can infer that the former option is a special case of the latter with $K=1$. In 
the following analysis, we will consider the general case and derive an expression for $R_{\max }$. For notation simplicity, we will replace $F_{k}$ by $F$ and $U_{k}$ by $U$ when we derive formulae for one precoding block.

Defining $P_{e_{i}}$ as the symbol error probability for a single transmission on a TU using the $i^{\text {th }}$ MCS, the overall probability of receiving an original symbol in error, denoted by $P_{e}$, can be expressed as:

$$
P_{e}=P_{e_{i}} \cdot \sum_{j=F-U}^{F-1}\left(\begin{array}{c}
F-1 \\
j
\end{array}\right) P_{e_{i}}^{j}\left(1-P_{e_{i}}\right)^{F-j-1}
$$

In words, an original symbol is received in error if symbol errors occur on both the TU carrying the original symbol and more than $F-U-1$ TUs from the remaining $F-1$ ones. Since error occurrences on individual TUs are independent of each other, we can use the central limit theorem (CLT) to approximate this probability as:

$$
P_{e} \approx P_{e_{i}} \cdot Q\left(\frac{\left(1-P_{e_{i}}\right)(F-1)-U}{\sqrt{(F-1)\left(1-P_{e_{i}}\right) P_{e_{i}}}}\right)
$$

Since the Q-function is a decreasing function with the increase of its argument, the lower $U$, the lower $P_{e}$. Thus, for any $i \in B$, we can reduce $U$ until the symbol error probability is reduced such that the corresponding bit error probability drops below the threshold bit error rate. We denote $U$ satisfying this condition on $F$ TUs using the $i^{\text {th }}$ MCS by $U_{i, F}$. To ensure that the bit error rate achieved by our scheme is always below the threshold bit error rate, we assume that if a symbol is received in error, all its bits are lost. Thus, defining $P_{e_{t}}$ as the maximum allowed symbol error probability for the $i^{\text {th }}$ MCS, then $P_{e_{t}}=P_{b}^{t h}$.

Using the CLT approximation defined in (2), we can calculate an approximate value of $U_{i, F}$ as follows:

$$
\begin{aligned}
& P_{e} \approx P_{e_{i}} \cdot Q\left(\frac{\left(1-P_{e_{i}}\right)(1-F)-U}{\sqrt{(F-1)\left(1-P_{e_{i}}\right) P_{e_{i}}}}\right) \leq P_{e_{t}} \\
& U_{i, F}^{\prime}=\left\lfloor\left(1-P_{e_{i}}\right)(F-1)\right. \\
&\left.\quad-\theta_{i} \sqrt{P_{e_{i}}\left(1-P_{e_{i}}\right)}(F-1)^{\frac{1}{2}}\right\rfloor \quad \forall F>1
\end{aligned}
$$

where $\theta_{i}=Q^{-1}\left(P_{e_{t}} / P_{e_{i}}\right)$. Note that $U_{i, F}$ should be upper bounded by $F-1$. Since in (3), $\theta_{i}$ can be negative for $P_{e_{i}} \in$ $\left(P_{e_{t}}, 2 P_{e_{t}}\right)$ which may lead to an increase in $U_{i, F}^{\prime}$ above its upper bound $F-1$, we can express $U_{i, F}$ as:

$$
U_{i, F}=\min \left\{U_{i, F}^{\prime}, F-1\right\}
$$

We can thus express the PRU throughput for the $i^{\text {th }}$ MCS as:

$$
R_{i}=b_{i} \sum_{k=1}^{K} U_{i, F_{k}}
$$

Proposition 1. For practical values of $N$ and $P_{e_{t}}$ such that $N \in\left\{1, \ldots, N_{u}\right\}$ where $N_{u}=1+\frac{1}{2 P_{e_{t}}}$, the maximum value for $R_{i}$ is achieved when $K=1$. In other words, $R_{i}^{\max }=$ $b_{i} U_{i, N}$.
To prove this proposition, we first need to introduce the following two lemmas. The proofs of these lemmas are found in the appendices.

Lemma 1. If $P_{e_{i}} \geq 2 P_{e_{t}}$, then $U_{i, F}$ is a superadditive function in $F$ on its domain $(F \geq 1)$.

Lemma 2. For $P_{e_{i}} \in\left(P_{e_{t}}, 2 P_{e_{t}}\right)$, if $F \leq F_{u}$, where $F_{u}=$ $1+\frac{1}{2 P_{e_{t}}}$, then $U_{i, F}$ is superadditive in $F \in\left\{1, \ldots, F_{u}\right\}$.

Proof of Proposition 1:

Since $F_{k} \leq N \leq 1+\frac{1}{2 P_{e_{t}}} \Rightarrow U_{i, F_{k}}$ is superadditive (from Lemmas 1 and 2). Thus

$$
\begin{aligned}
R_{i}\{K=1\} & =b_{i} U_{i, N} \\
& =b_{i} U_{i, \sum_{k=1}^{K} F_{k}} \quad \forall K>1 \\
& \geq b_{i} \sum_{k=1}^{K} U_{i, F_{k}} \quad \forall K>1 \\
& =R_{i}\{K>1\}
\end{aligned}
$$

Thus, we are always sure that "no partitioning" results in the maximum value of $R_{i}$ and that $R_{i}^{\max }=b_{i} U_{i, N}$.

From Proposition 1, the maximum PRU throughput is:

$$
R_{\max }=\max _{i \in B}\left\{b_{i} U_{i, N}\right\}
$$

\section{B. Improvement Conditions}

Defining $R_{c}$ as the throughput achieved by the conventional technique, an improvement in the PRU throughput is obtained by our precoding scheme over the conventional one iff $R_{\max }>R_{c}$. That occurs iff:

$$
\exists i \in B: \quad U_{i, N} b_{i}>N b_{c} \Rightarrow U_{i, N}>\frac{N b_{c}}{b_{i}}
$$

Defining $\beta_{i}=b_{c} / b_{i}, \alpha_{i}=\theta_{i} \sqrt{P_{e_{i}}\left(1-P_{e_{i}}\right)}$ and $\nu_{i}=$ $1-P_{e_{i}}-\beta_{i}$, the following proposition provides sufficient conditions on the range of $N$ to guarantee the satisfaction of the improvement condition in (7)

Proposition 2. For practical values of $N$ and $P_{e_{t}}$ such that $N \leq N_{u}$, a throughput improvement is achieved by our proposed technique (i.e. $R_{\max }>R_{c}$ ) if $\nu_{i}>0$ and if:

$$
N> \begin{cases}\frac{2}{1-\beta_{i}} & P_{e_{i}} \in\left(P_{e_{t}}, 2 P_{e_{t}}\right) \\ 1+\left(\frac{\alpha_{i}+\sqrt{\alpha_{i}^{2}+4 \nu_{i}\left(1+\beta_{i}\right)}}{2 \nu_{i}}\right)^{2} & P_{e_{i}} \geq 2 P_{e_{t}}\end{cases}
$$

Proof:

Case 1: $P_{e_{i}} \in\left(P_{e_{t}}, 2 P_{e_{t}}\right)$

If $N>2\left(1-\beta_{i}\right)^{-1} \Rightarrow N-2>N \beta_{i}$. But since $N \leq N_{u} \Rightarrow U_{i, N} \geq N-2$ (from proof of Lemma 2), then $U_{i, N}>N \beta_{i}$. 
TABLE I

NUMERICAL VALUES OF $\theta_{i}, \nu_{i}$ AND $N_{i_{\text {min }}}$ FOR $P_{b}^{t h}=10^{-3}, 10^{-4}$ AND DIFFERENT VALUES OF SINR

\begin{tabular}{|c|c|c|c|c|c|c|}
\hline$P_{b}^{t h}$ & SINR & $b_{c}$ & $b_{i}$ & $\theta_{i}$ & $\nu_{i}$ & $N_{i_{\min }}$ \\
\hline \hline $10^{-3}$ & $7 \mathrm{~dB}$ & 2 & 4 & 1.8849 & 0.4664 & 6 \\
& & & 6 & 2.4245 & 0.5362 & 8 \\
& & & 8 & 2.6046 & 0.5326 & 10 \\
\hline $10^{-3}$ & $15 \mathrm{~dB}$ & 4 & 6 & -0.3786 & 0.3318 & 7 \\
& & & 8 & 1.9515 & 0.4608 & 7 \\
\hline $10^{-3}$ & $20 \mathrm{~dB}$ & 6 & 8 & -2.3170 & 0.2490 & 9 \\
\hline \hline $10^{-4}$ & $10 \mathrm{~dB}$ & 2 & 4 & 1.9029 & 0.4965 & 5 \\
& & & 6 & 2.8927 & 0.6143 & 6 \\
& & & 8 & 3.2079 & 0.6004 & 9 \\
\hline $10^{-4}$ & $15 \mathrm{~dB}$ & 4 & 6 & 1.5161 & 0.3318 & 7 \\
& & & 8 & 2.8006 & 0.4608 & 8 \\
\hline $10^{-4}$ & $20 \mathrm{~dB}$ & 6 & 8 & 1.2874 & 0.2490 & 9 \\
\hline
\end{tabular}

Case 2: $P_{e_{i}} \geq 2 P_{e_{t}}$

If the condition holds, then:

$$
(N-1)^{\frac{1}{2}}>\frac{\alpha_{i}+\sqrt{\alpha_{i}^{2}+4 \nu_{i}\left(1+\beta_{i}\right)}}{2 \nu_{i}}
$$

Since $\nu_{i}>0$

$$
\begin{aligned}
& \text { Thus } \nu_{i}(N-1)-\alpha_{i}(N-1)^{\frac{1}{2}}-\left(1+\beta_{i}\right)>0 \\
& \Rightarrow \hat{U}_{i, N}>N \beta_{i}+1 \Rightarrow\left\lfloor\hat{U}_{i, N}\right\rfloor>N \beta_{i} \\
& \Rightarrow U_{i, N}>N \beta_{i}
\end{aligned}
$$

In the following section, we will show that the practical PRU sizes (ranging from 80 to 400) satisfy these sufficient conditions for different system settings.

\section{Numerical EVALUATION}

In this numerical evaluation, uncoded modulations were employed. The allowed constellations are (BPSK, QPSK, 16QAM, 64-QAM, 256-QAM) corresponding to spectral efficiencies of $(1,2,4,6,8)$, respectively. The value for $P_{b}^{t h}$ is set to $10^{-3}$ or $10^{-4}$ for which $N_{u}>501,1001$ TUs respectively. Since practical PRU sizes range from 80 to 400 TUs, the upper bound set by Propositions 1 and 2 is already satisfied.

Defining $N_{i_{m i n}}$ as the smallest PRU size satisfying the sufficient conditions provided in Proposition 2, Table I depicts the values of $\theta_{i}, \nu_{i}$ and $N_{i_{m i n}}$ for $P_{b}^{t h}=10^{-3}$ and $10^{-4}$ and for different SINR values.

We can clearly observe that the values of $\nu_{i}$ are always positive and that the values of $N_{i_{\text {min }}}$ are very low compared to practical PRU sizes that range from 80 to 400 TUs. This shows that our proposed technique guarantees throughput improvement in practical systems.

For $P_{b}^{t h}=10^{-3}$, Figure 1 depicts a comparison between the throughput performance of the conventional and precoding schemes against SINR for $N=200$ TUs. Figure 2 depicts the same comparison against the PRU size $(N)$ for $\mathrm{SINR}=17$ $\mathrm{dB}$. In both figures, the percentage increase of the precoding scheme over the conventional one is also illustrated. The
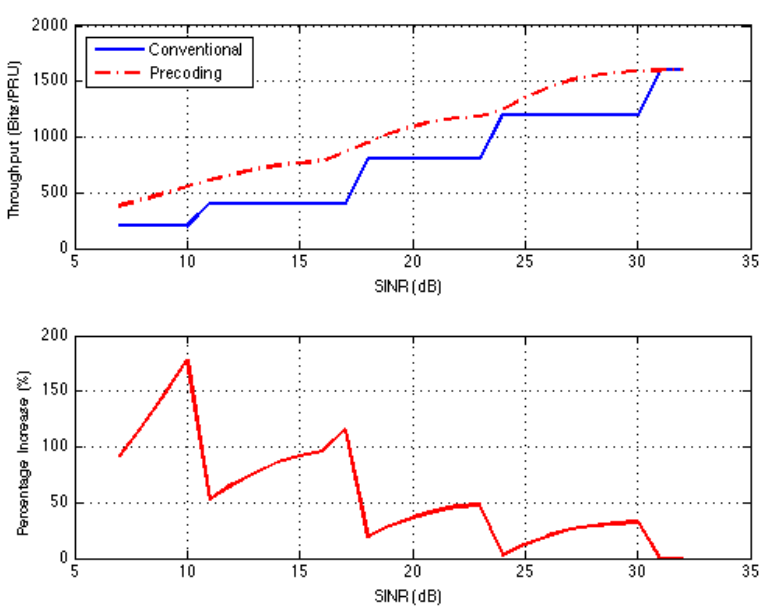

Fig. 1. Comparison between $R_{c}$ and $R_{\max }$ against SINR for $N=200$ TUs and $P_{b}^{t h}=10^{-3}$.
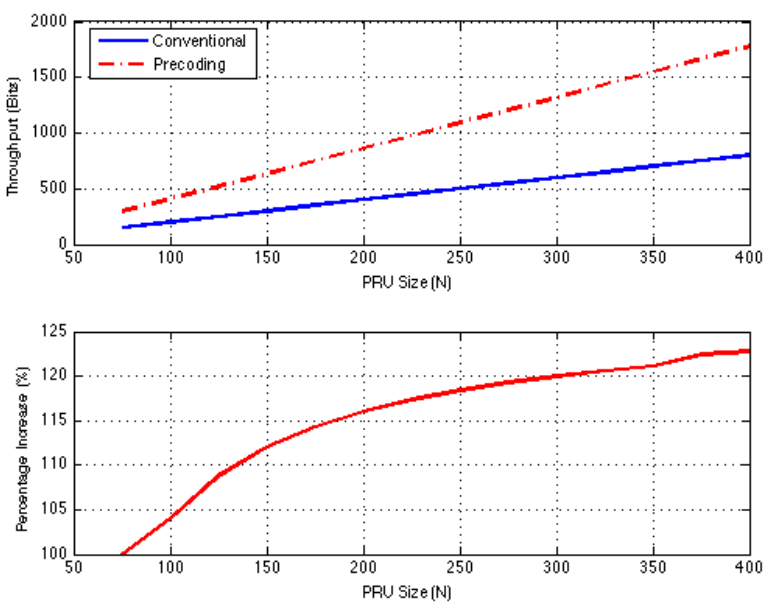

Fig. 2. Comparison between $R_{c}$ and $R_{\max }$ against $N$ for $\operatorname{SINR}=17 \mathrm{~dB}$ and $P_{b}^{t h}=10^{-3}$

percentage increase is defined as:

$$
\text { Percentage Increase }=\frac{R_{\max }-R_{c}}{R_{c}} \times 100
$$

From both figures, we can observe that a throughput improvement is obtained by our proposed technique for different SINR values and different PRU sizes for the given settings. Similar improvements are also achievable for other combinations of $N$, SINR and $P_{b}^{t h}$. We omitted these results due to space limitations.

\section{CONCLUSION}

In this paper, we proposed the use of the precoding scheme, we suggested in [1], to increase the MCS level employed in the PRUs of OFDMA based wireless systems with limited feedback. We derived a formula for the number of original distinct data symbols that should be coded in each PRU as 
well as the sufficient conditions for our proposed scheme to outperform the conventional scheme. Our analysis and numerical evaluations showed that this technique can indeed achieve a considerable increase in the PRU throughput when the conventional technique fails in employing the highest MCS in this PRU.

\section{REFERENCES}

[1] A. Yazdi, S. Sorour, S. Valaee, and R. Kim, "Reducing symbol loss probability in the downlink of an ofdma based wireless network," IEEE International Conference on Communications (ICC'08)., pp. 3485-3489, May 2008.

[2] Air Interface for Fixed and Mobile Broadband Wireless Access Systems - Amendment for Physical and Medium Access Control Layers for Combined Fixed and Mobile Operation in Licensed Bands, IEEE Standard for Local and Metropolitan Area Networks - Part 16 (IEEE 802.16e) Std. IEEE 802.16e/D8-2005, May 2005.

[3] Technical Specification Group Radio Access Network - Physical layer aspects for evolved Universal Terrestrial Radio Access (UTRA) (Release 7), 3rd Generation Partnership Project (3GPP-LTE) Std. 3GPP-LTE TR 25.814 V7.0.0, 2006.

[4] C. Y. Wong, R. Cheng, K. Lataief, and R. Murch, "Multiuser ofdm with adaptive subcarrier, bit, and power allocation," IEEE Journal on Selected Areas in Communications, vol. 17, no. 10, pp. 1747-1758, Oct 1999.

[5] C. Y. Wong, C. Tsui, R. Cheng, and K. Letaief, "A real-time sub-carrier allocation scheme for multiple access downlink ofdm transmission," IEEE Vehicular Technology Conference (VTC'99-Fall), vol. 2, pp. 11241128 vol.2, 1999.

[6] W. Rhee and J. Cioffi, "Increase in capacity of multiuser ofdm system using dynamic subchannel allocation," IEEE Vehicular Technology Conference (VTC'00-Spring), vol. 2, pp. 1085-1089 vol.2, 2000.

[7] S. Pietrzyk and G. Janssen, "Multiuser subcarrier allocation for qos provision in the ofdma systems," IEEE Vehicular Technology Conference (VTC'02-Fall), vol. 2, pp. 1077-1081 vol.2, 2002.

[8] Y. J. Zhang and K. Letaief, "Multiuser adaptive subcarrier-and-bit allocation with adaptive cell selection for ofdm systems," IEEE Transactions on Wireless Communications, vol. 3, no. 5, pp. 1566-1575, Sept. 2004.

[9] Z. Wang and G. Giannakis, "Linearly precoded or coded ofdm against wireless channel fades?" IEEE Third Workshop on Signal Processing Advances in Wireless Communications (SPAWC'01), pp. 267-270, 2001.

[10] Y. Yu, R. Lin, and A. Petropulu, "Linearly precoded ofdm system with adaptive modulation," IEEE International Conference on Acoustics, Speech, and Signal Processing (ICASSP'04), vol. 2, pp. ii-417-20 vol.2, May 2004.

[11] A. A. Yazdi, "Optimum packet loss rate and peak-to-average power ratio reduction in multicarrier networks," Master's thesis, Dept. of Electrical and Computer Engineering, University of Toronto, 2008.

\section{APPENDix A \\ PROOF OF LEMMA 1}

as:

If $P_{e_{i}} \geq 2 P_{e_{t}} \Rightarrow \theta_{i} \geq 0 \Rightarrow U_{i, F}=U_{i, F}^{\prime}$. Define $\hat{U}_{i, F}$

$$
\hat{U}_{i, F}=\left(1-P_{e_{i}}\right)(F-1)-\theta_{i} \sqrt{P_{e_{i}}\left(1-P_{e_{i}}\right)}(F-1)^{\frac{1}{2}}
$$

Clearly, $U_{i, F}=\left|\hat{U}_{i, F}\right|$. The superadditivity of $\hat{U}_{i, F}$ follows from the linearity and increasing monotonicity of its first term and the subadditivity of its second term since $\theta_{i} \geq 0$. Thus, we have:

$$
\begin{aligned}
& \left\lfloor\hat{U}_{i, F_{1}+F_{2}}\right\rfloor \geq\left\lfloor\left(\hat{U}_{i, F_{1}}+\hat{U}_{i, F_{2}}\right)\right\rfloor \geq\left\lfloor\hat{U}_{i, F_{1}}\right\rfloor+\left\lfloor\hat{U}_{i, F_{2}}\right\rfloor \\
\Rightarrow & U_{i, F_{1}+F_{2}} \geq U_{i, F_{1}}+U_{i, F_{2}} .
\end{aligned}
$$

\section{APPENDIX B \\ PROOF OF LEMMA 2}

For $P_{e_{i}} \in\left(P_{e_{t}}, 2 P_{e_{t}}\right), \theta_{i}<0$. So we can re-write the expression for $U_{i, F}^{\prime}$ in (3) as follows:

$$
\begin{aligned}
U_{i, F}^{\prime}= & \left\lfloor\left(1-P_{e_{i}}\right)(F-1)\right. \\
& \left.+\left|\theta_{i}\right| \cdot \sqrt{P_{e_{i}}\left(1-P_{e_{i}}\right)}(F-1)^{\frac{1}{2}}\right\rfloor
\end{aligned}
$$

Thus, for any value of $F>1$,

$$
\begin{aligned}
\inf _{P_{e_{i}} \in\left(P_{e_{t}}, 2 P_{e_{t}}\right)} U_{i, F}^{\prime}= & \inf _{P_{e_{i}} \in\left(P_{e_{t}}, 2 P_{e_{t}}\right)}\left\{\left\lfloor\left(1-P_{e_{i}}\right)(F-1)\right.\right. \\
& \left.\left.+\left|\theta_{i}\right| \sqrt{P_{e_{i}}\left(1-P_{e_{i}}\right)}(F-1)^{\frac{1}{2}}\right\rfloor\right\} \\
= & \left\lfloor\operatorname { i n f } _ { P _ { e _ { i } } \in ( P _ { e _ { t } } , 2 P _ { e _ { t } } ) } \left\{\left(1-P_{e_{i}}\right)(F-1)\right.\right. \\
& \left.\left.+\left|\theta_{i}\right| \cdot \sqrt{P_{e_{i}}\left(1-P_{e_{i}}\right)}(F-1)^{\frac{1}{2}}\right\}\right\rfloor \\
= & \left\lfloor\left(1-2 P_{e_{t}}\right)(F-1)\right\rfloor
\end{aligned}
$$

Since $F \leq 1+\frac{1}{2 P_{e_{t}}} \Rightarrow P_{e_{t}} \leq \frac{1}{2(F-1)}$, then

$$
\inf _{P_{e_{i}} \in\left(P_{e_{t}}, 2 P_{e_{t}}\right)} U_{i, F}^{\prime}=\lfloor F-2\rfloor=F-2
$$

Thus, for any value of $P_{e_{i}} \in\left(P_{e_{t}}, 2 P_{e_{t}}\right)$, and $F \in$ $\left\{2, \ldots, F_{u}\right\}, U_{i, F}^{\prime} \geq F-2$. Substituting in (4), we can clearly see that $U_{i, F}$ can only be $F-2$ or $F-1$. The change from the first to the second formula occurs when:

$$
U_{i, F}^{\prime}=F-1 \Rightarrow F \leq 1+\frac{\theta_{i}^{2}\left(1-P_{e_{i}}\right)}{P_{e_{i}}}
$$

For any value of the right-hand-side of the above inequality and $F \in\left\{2, \ldots, F_{u}\right\}$, we can only have four possible cases:

1) If $U_{i, F_{1}}=U_{i, F_{2}}=U_{i, F_{1}+F_{2}}=F-1$ :

$$
U_{i, F_{1}+F_{2}}-\left(U_{i, F_{1}}+U_{i, F_{2}}\right)=1 \geq 0 ;
$$

2) If $U_{i, F_{1}}=U_{i, F_{2}}=F-1$ and $U_{i, F_{1}+F_{2}}=F-2$ :

$$
U_{i, F_{1}+F_{2}}-\left(U_{i, F_{1}}+U_{i, F_{2}}\right)=0 \geq 0 ;
$$

3) If $U_{i, F_{1}}=F-1$ and $U_{i, F_{2}}=U_{i, F_{1}+F_{2}}=F-2$ :

$$
U_{i, F_{1}+F_{2}}-\left(U_{i, F_{1}}+U_{i, F_{2}}\right)=1 \geq 0 ;
$$

4) If $U_{i, F_{1}}=U_{i, F_{2}}=U_{i, F_{1}+F_{2}}=F-2$ :

$$
U_{i, F_{1}+F_{2}}-\left(U_{i, F_{1}}+U_{i, F_{2}}\right)=2 \geq 0 .
$$

Thus, $U_{i, F}$ is superadditive for the four possibilities $\Rightarrow$ superadditive in $F \in\left\{2, \ldots, F_{u}\right\}$. Finally, for $F=1, U_{i, F}=0$ which maintains the superadditivity of the function in the range $F \in\left\{1, \ldots, F_{u}\right\}$. This concludes the proof. 